\title{
Measuring the breathless brain: is real life too noisy?
}

\author{
Andrew Binks
}

Affiliation: Biomedical Sciences, School of Medicine Greenville, University of South Carolina, Greenville, SC, USA.

Correspondence: Andrew Binks, School of Medicine, Greenville, University of South Carolina, Biomedical Sciences, 701 Grove Road Health Sciences Administration Building, Greenville, SC 29605, USA.

E-mail: BINKSAgreenvillemed.sc.edu

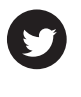

@ERSpublications

The first brain study of "real-life" dyspnoea is limited by real-life, but gives us a renewed sense of direction http://ow.ly/T70dp

In our 15-year history of mapping the central neural mechanisms of dyspnoea, we have discovered the complexities of obtaining an objective measure of the sensation and begun discriminating the emotional, cognitive and behavioural responses to it. The task has been complicated by use of a variety of laboratory models that likely produce varying forms of dyspnoea. There are at least three neurally distinct forms (air hunger, effort to breathe and chest tightness [1]) and each form has quantitatively, and maybe qualitatively, different affective components [2]. The stimuli used to gain the first insights into the "breathless brain" were tidal volume restriction [3], hypercapnia [4] and resistance loaded breathing [5]. The most consistent finding in these and subsequent studies [6-8] is activation of the insula cortex; a region also involved with the perception of other homeostatic warning signals such as pain, hunger and thirst [9]. The involvement (or not) of other brain regions has varied between studies and likely reflects the variety of stimuli and types of dyspnoea they generated. The most consistent factor throughout these studies is the use of healthy individuals as subjects.

After achieving a variety of neural signatures for dyspnoea using lab models, it seems timely to determine the neural signature of "real-life" dyspnoea in patients. Published in this issue, Higashimoto et al. [10] have been the first to investigate the neural activity of "real-life" dyspnoea in chronic obstructive pulmonary disease (COPD) patients with the "real-life" stimulus of exercise. But as much as marking a new era of study, the paper led me to re-assess what we should be looking for as the investigation of dyspnoea's central mechanisms moves forward. I was once asked, at a scientific meeting, "why we were doing this, and how is this ever going to help my patients?" As we vigorously search for all sorts of biomarkers of all types of pathologies it seemed strange that the search for an objective measure of the major symptom of lung and heart disease was questionable. Regardless, our current subjective measurements of dyspnoea include multiple iterations of simple scales where a subject choses between 1 and 10. An objective measurement would remove inter-personal variability (e.g. one person's 5 may be worse than another person's 8), subject/ patient bias and patient/subject misinterpretation of what we wished them to rate. Maybe the questioner thought it a merely academic pursuit to study dyspnoea's neural circuitry, because, even when our dyspnoea map is complete there is no therapy to relieve it ("...how is this ever going to help my patients?"). The obvious argument is that knowledge of dyspnoea's neural circuitry will focus the development of therapies, and be able to objectively test their efficacy.

So what should our short- and long-term goals for studying the breathless brains of patients be? In the short-term the comparison of the "real-life" patient responses to those seen in 15 years of laboratory-induced dyspnoea will allow us to assess the pertinence of our laboratory models and validate or reject their continued use. For the long term we need to unravel the sensory, emotional and behavioural

Received: Aug 312015 | Accepted: Sept 272015

Conflict of interest: None declared.

Copyright OERS 2015 
components [11] of "real-life" dyspnoea to focus the development and assessment of therapies. So have Higashimoto et al. [10] taken us closer to achieving these goals?

The study by Higashimoto et al. [10] provides a very limited comparison with previous lab-based models and the restricted view is the ironic cost of generating "real-life" dyspnoea. The exercise-based protocol led the authors to reject more comprehensive brain imaging techniques, such as functional magnetic resonance imaging (fMRI) and positron emission tomography (PET). Near-infrared spectroscopy can tolerate exercise related head movement, but its field of view is limited to cortical features. While it is appreciably more difficult to perform an exercise-based protocol with PET or fMRI, it is not impossible. A recent exercise study used fMRI to show activations with perceived exertion in healthy individuals [12], and a PET study [13] has shown an insula response during exercise. Although these studies did not measure dyspnoea per se, they've demonstrated proof of concept. I'd argue that because a lower intensity of exercise is needed to induce dyspnoea in COPD patients, the problem of exercise related head-movement would be less in patients than it was in the healthy individuals used in these studies. However, in the report by HigASHIMOTO et al. [10], the pre-motor cortex (PMA) activation is consistent with laboratory dyspnoea induced by tidal volume reduction [7] and resistance-loaded breathing [14]. But, none of these studies can determine whether the PMA activation is integral to dyspnoea itself, or part of the downstream response to it.

The second issue with the Higashimoto et al. [10] "real-life" dyspnoea is that exercise is a very noisy stimulus that not only involves dyspnoea-related activity, but also numerous central activations [15] and afferent signals generated by the exercise itself. As exercise becomes more intense the exercise-related neural activity increases, as does that associated with dyspnoea. Parallel neural changes are difficult to attribute to one response or another; a problem acknowledged by the authors. A similar analytical problem has arisen with previous dyspnoea models, where dyspnoea-related neural activity is paralleled by activity related to the consequent emotional responses (e.g. anxiety) and, therefore, they become difficult to distinguish. Only now, 15 years after the first studies, are these beginning to be parsed apart $[7,16]$. To fulfil the long-term goals, we not only have to distinguish and measure different forms of dyspnoea, but also the emotional responses to them, as identifying distinct (but connected) networks may provide distinct therapeutic opportunities.

Determining the emotional and sensory responses of patients will be particularly important, as they are very likely to be different to those observed in healthy individuals [11]. Healthy individuals undergoing lab-based models of dyspnoea know that the sensation will stop when the study does, while the COPD patients' chronic exposure to "real-life" dyspnoea might prevent that reassurance; merely thinking about situations that cause dyspnoea generates an emotional response in patients [16]. Chronic exposure to dyspnoea might also result in either sensitisation [17] or adaptation [18] of the sensory and/or emotional components and produce a very different neural signature to those we see in healthy individuals. The different forms of dyspnoea experienced across different diseases [19] are likely to have different neural signatures, each being constructed of a combination of their own sensory and emotional elements. Addressing these differences in patients will be important for the appropriate application of future therapies to target specific areas. For example, providing anxiolytics for patients with psychogenic dyspnoea is effective in resolving the emotional origin and the resultant dyspnoea, but this approach hasn't been effective for patients with dyspnoea with physical origins [20]. Morphine may reduce both the sensory and affective components of air hunger [21], but this should be objectively tested.

In summary, HigasHimoto et al. [10] have taken an important first step in gaining an understanding of the neural signature of dyspnoea in COPD. Continuing this path with more comprehensive studies and including other diseases will help us refine our laboratory models to confidently and more accurately mimic the dyspnoea of specific disease states. To do this, we will have to overcome our disparate origins in pulmonology, cardiology, palliative care, nursing and psychiatry, and the field of dyspnoea research has to use the same language to ask the same questions to probe the multiple forms and levels of dyspnoea in different patients $[11,22,23]$. Once the laboratory models are established we can make controlled and cleaner interventions and avoid the noise and vagaries of the clinical setting and the technical and analytical problems of "real-life" exercise-based protocols.

\section{References}

1 Parshall MB, Schwartzstein RM, Adams L, et al. An official American Thoracic Society statement: update on the mechanisms, assessment, and management of dyspnea. Am J Respir Crit Care Med 2012; 185: 435-452.

2 Banzett RB, Pedersen SH, Schwartzstein RM, et al. The affective dimension of laboratory dyspnea: air hunger is more unpleasant than work/effort. Am J Respir Crit Care Med 2008; 177: 1384-1390.

3 Banzett RB, Mulnier HE, Murphy K, et al. Breathlessness in humans activates insular cortex. Neuroreport 2000; 11: $2117-2120$.

4 Brannan S, Liotti M, Egan G, et al. Neuroimaging of cerebral activations and deactivations associated with hypercapnia and hunger for air. Proc Natl Acad Sci USA 2001; 98: 2029-2034.

5 Peiffer C, Poline JB, Thivard L, et al. Neural substrates for the perception of acutely induced dyspnea. Am J Respir Crit Care Med 2001; 163: 951-957. 
Evans KC, Banzett RB, Adams L, et al. BOLD fMRI identifies limbic, paralimbic, and cerebellar activation during air hunger. J Neurophysiol 2002; 88: 1500-1511.

7 Binks AP, Evans KC, Reed JD, et al. The time-course of cortico-limbic neural responses to air hunger. Respir Physiol Neurobiol 2014; 204: 78-85.

8 Schon D, Rosenkranz M, Regelsberger J, et al. Reduced perception of dyspnea and pain after right insular cortex lesions. Am J Respir Crit Care Med 2008; 178: 1173-1179.

9 Denton DA, McKinley MJ, Farrell M, et al. The role of primordial emotions in the evolutionary origin of consciousness. Conscious Cogn 2009; 18: 500-514.

10 Higashimoto Y, Honda N, Yamagata T, et al. Exertional dyspnoea and cortical oxygenation in patients with COPD. Eur Respir J 2015; 46: 1615-1624.

11 Laviolette L, Laveneziana P. Dyspnoea: a multidimensional and multidisciplinary approach. Eur Respir J 2014; 43: $1750-1762$.

12 Fontes EB, Okano $\mathrm{AH}$, De Guio $\mathrm{F}$, et al. Brain activity and perceived exertion during cycling exercise: an fMRI study. Br J Sports Med 2015; 49: 556-560.

13 Hiura M, Nariai T, Ishii K, et al. Changes in cerebral blood flow during steady-state cycling exercise: a study using oxygen-15-labeled water with PET. J Cereb Blood Flow Metab 2014; 34: 389-396.

14 Peiffer C, Costes N, Herve P, et al. Relief of dyspnea involves a characteristic brain activation and a specific quality of sensation. Am J Respir Crit Care Med 2008; 177: 440-449.

15 Christensen LO, Johannsen P, Sinkjaer T, et al. Cerebral activation during bicycle movements in man. Exp Brain Res 2000; 135: 66-72.

16 Herigstad M, Hayen A, Evans E, et al. Dyspnea-related cues engage the prefrontal cortex: evidence from functional brain imaging in COPD. Chest 2015: 148; 953-961.

17 Stoeckel MC, Esser RW, Gamer M, et al. Brain mechanisms of short-term habituation and sensitization toward dyspnea. Front Psychol 2015; 6: 748.

18 Carrieri-Kohlman V, Douglas MK, Gormley JM, et al. Desensitization and guided mastery: treatment approaches for the management of dyspnea. Heart Lung 1993; 22: 226-234.

19 Simon PM, Schwartzstein RM, Weiss JW, et al. Distinguishable types of dyspnea in patients with shortness of breath. Am Rev Respir Dis 1990; 142: 1009-1014.

20 Thomas JR, von Gunten CF. Management of dyspnea. J Support Oncol 2003; 1: 23-32

21 Banzett RB, Adams L, O’Donnell CR, et al. Using laboratory models to test treatment: morphine reduces dyspnea and hypercapnic ventilatory response. Am J Respir Crit Care Med 2011; 184: 920-927.

22 Banzett RB, O’Donnell CR, Guilfoyle TE, et al. Multidimensional Dyspnea Profile: an instrument for clinical and laboratory research. Eur Respir J 2015; 45: 1681-1691.

23 Banzett RB, O’Donnell CR. Should we measure dyspnoea in everyone? Eur Respir J 2014; 43: 1547-1550. 